\title{
The PC tachistoscope has 32 pages
}

\author{
BRETT MYORS \\ Macquarie University, Sydney, New South Wales, Australia
}

\begin{abstract}
Page switching is the basis of the $\mathrm{PC}$ tachistoscope, and usual accounts of page switching on the $\mathrm{PC}$ refer to a maximum of 8 available pages. This article shows how to achieve up to 16 pages in 40 -column text mode and up to 32 pages in low-resolution graphics mode using conventional page switching combined with the bit-plane layering technique described by Bokhorst (1995). A high level C program is presented to show how this is done. When 32 pages are in use, the images must be monochrome.
\end{abstract}

Segalowitz (1987) was one of the first authors to describe how the PC could be used for tachistoscopic presentation of stimuli by preloading images into various pages of video memory and rapidly switching between them. In this way, images did not need to be drawn in real time and could therefore be displayed at the maximum possible rate simply by instructing the video hardware to switch to a new image stored at a different location in memory. The maximum display rate is governed by the screen refresh rate of the video monitor-which is typically around $60-70 \mathrm{~Hz}$, although it can vary anywhere between 40 and $90 \mathrm{~Hz}$ (Haussmann, 1992). EGA and VGA video standards provide well-documented support for 8 pages in text modes and up to 8 pages in graphics modes, and many authors have described how to access these (see Haussmann, 1992, for a review). However, the number of available pages can be substantially increased relatively easily, thus increasing the capacity and usefulness of the PC tachistoscope. The purpose of this article is to show how the maximum number of available pages can be increased to 16 pages in text mode and 32 in graphics mode.

\section{THE MODE 01h TACHISTOSCOPE}

Mode $01 \mathrm{~h}$ is a 40 -column $\times 25$-line text mode available on the original IBM PC and supported in all subsequent models; thus, it is universally available on PCs with at least CGA capabilities. It is well known that mode $01 \mathrm{~h}$ supports 8 separate text screens, but because of its low memory requirements, this limit can be easily doubled. In text modes, a screen of display memory comprises many 2-byte character/attribute pairs, and only 1,000 of these are required per screen in mode $01 \mathrm{~h}$. Table 1 shows the starting addresses of the pages available in mode $01 \mathrm{~h}$. Characters and attributes can be written directly to video memory at appropriate offsets from these locations and subsequently displayed simply by changing the start address registers of the CRT Controller. Modifying the start address

Correspondence concerning this article should be addressed to B. Myors, School of Behavioural Sciences, Macquarie University, Sydney, NSW 2109, Australia (e-mail: bmyors@bunyip.bhs.mq.edu.au). registers changes the origin of the next screen update and hence changes the display. The lines indicated by two asterisks in the set_page() routine in Listing 1 show the $\mathrm{C}$ code for making these changes. Accessing the CRT Controller directly allows the number of available screens for display to be increased from 8 to 16 .

\section{THE MODE Odh TACHISTOSCOPE}

Even more pages are available with the mode $0 \mathrm{dh}$ tachistoscope. Mode $0 \mathrm{dh}$ is a graphics mode with $320 \times$ 200 pixel resolution, introduced with the EGA and upwardly compatible to the VGA. Mode Odh is a planar mode with display memory organized into four bit-planes used to access any one of 16 possible colors. Images are therefore spread across the four planes, resulting in a somewhat complex method of addressing a single pixel (see Farraro, 1994, or Wilton, 1994, for details). Because of its comparatively low resolution, mode $0 \mathrm{dh}$ also supports 8 conventional screen pages; however, if the bit-plane layering technique described by Bokhorst (1995) is used in conjunction with the usual page switching described above, the number of available pages is effectively quadrupled. Thus there are 8 conventional display pages, each of which comprises four separate images stored in each bit-plane. Any of these images can be individually accessed and displayed. Although the resolution of mode $0 \mathrm{dh}$ is low, it is quite effective for simple graphics and large text.

Listing 1 shows a $\mathrm{C}$ program that demonstrates the 32 page mode 0dh tachistoscope. The program uses Microsoft $\mathrm{C}$ graphics routines to change video modes and fills each page with an ASCII character, starting with a capital A on page 0 and ending with a single quote on page 31 . Character strings are written by directly accessing bitmaps for the $8 \times 8$ character set stored in ROM. Fortunately these bitmaps are compatible with planar memory modes and make text drawing relatively easy. The user is then able to switch between pages with the up or down arrow keys.

When the user decides to exit, the program automatically performs $1,000,000$ page switches and prints out the elapsed time. Times are measured by the standard $\operatorname{clock}()$ function, which uses the on-board time-of-day clock. As 
Table 1 Screen Pages in Mode $01 \mathrm{~h}$

\begin{tabular}{cc} 
Page & Start Address \\
\hline 0 & b8000000h \\
1 & b8000800h \\
2 & b8001000h \\
4 & b8001800h \\
5 & b8002000h \\
6 & b8002800h \\
7 & b8003000h \\
8 & b8003800h \\
9 & b8004000h \\
10 & b8004800h \\
11 & b8005000h \\
12 & b8005800h \\
13 & b8006000h \\
14 & b8006800h \\
15 & b8007000h \\
\hline
\end{tabular}

Note-The first 8 pages are well documented but the subsequent 8 pages are also usually available.

such, clock () is a low-resolution timer with a period of only $54.9 \mathrm{msec}$ - hence the use of $1,000,000$ trials to gain adequate accuracy.

The enable_plane() function shows how the Map Mask Register of the VGA Sequencer can be set to allow only one plane to be written to at a time. Page switching is performed by the set_page() function, which shows how the Color Plane Enable Register of the Attribute Controller can be used to select an individual bit-plane for display. Use of the start address registers of the CRT Controller for conventional page switching has already been described. The function sync() shows how to lock onto the vertical retrace via the Input Status Register. Locking onto the vertical retrace ensures that screen changes occur only while the electron guns in the monitor are moving from the bottom right-hand corner of the screen to the top left-hand corner and is essential for tachistoscopic applications and smooth screen transitions. Ideally the code for sync() would be incorporated into set_page(), to avoid an unnecessary function call, but it was kept separate so that set_page() could be accurately timed.

The program shown in Listing 1 provides all the relevant display routines for tachistoscopic presentation of text stimuli, including how to display a text string at an arbitrary location on the screen. Resolution of the $8 \times 8$ character set is surprisingly good in this mode. One likely additional requirement, not shown, is the setting of VGA color registers $1,2,4$, and 8 to the same color. As it stands, the demonstration program leaves the default colors unchanged, so that screens involving plane 1 are shown in blue, screens involving plane 2 are shown in green, screens involving plane 4 are shown in red, and screens involving plane 8 are displayed in dark gray. The color registers can easily be altered with a BIOS call. If graphical stimuli such as simple geometric shapes are required, new functions will need to be written in order to load them appropriately.
One limitation of the current scheme is that the images must all be monochrome because only one bit-plane is used for each image. Multicolor images could be set up by utilizing more than one bit-plane per image, but this would reduce the number of planes available for layering and hence the number of display pages.

\section{DISCUSSION}

Bokhorst (1995) provided assembly language routines for bit-plane layering in modes $10 \mathrm{~h}$ and $12 \mathrm{~h}$. Mode $12 \mathrm{~h}$ does not support any conventional pages at all, and mode $10 \mathrm{~h}$ only supports two, giving a maximum of 8 pages available when layering is used. In contrast, the techniques described here support considerably more than this.

Results of the timing of the set_page() function in Listing 1 were periods of $5.44 \mu \mathrm{sec}$ for a single page switch on a Pentium 100 computer with an STB Powergraph 64 video accelerator card. This is an order of magnitude less than the retrace interval of $60 \mu \mathrm{sec}$ (Sargent \& Shoemaker, 1995) and indicates that this computer is suitable for tachistoscopic operations using the methods described here. Comparable results were obtained on other computers tested.

Areas of substantive interest where the techniques described might prove useful include the rapid sequential visual presentation (RSVP) paradigm (Forster, 1970; Potter, 1993) used to study very short-term conceptual memory, and the inspection time paradigm used in cognitive abilities studies (Nettlebeck, 1987). In particular, the RSVP paradigm involves a large number of images. For example, discovery of the attentional blink (Raymond, Shapiro, \& Arnell, 1992) involved up to 24 images, well in excess of the conventional PC tachistoscope.

Of course, if a new image can be written to display memory inside the duration of a single vertical retrace, the number of pages available to the PC tachistoscope is effectively infinite. However, the retrace interval is extremely short. Precise timing with the mode 2 timer (Graves \& Bradley, 1991) showed that it takes about $0.2 \mathrm{msec}$ to completely fill the mode $01 \mathrm{~h}$ screen on the Pentium 100 PC. ${ }^{1}$ Thus, only about $30 \%$ of the image can be drawn within the retrace interval, and it is unlikely that any other video mode will improve on the performance of this lowresolution $40 \times 25$ line text mode. We are left with the conventional method of preloading images into display memory and switching between them.

As a matter of caution, it is recommended that tachistoscopic and other precision timing operations be carried out in the native DOS environment only, since only oldfashioned DOS guarantees complete, uninterrupted control of the PC hardware at the lowest level. Windows, Windows ' 95 , or any other multitasking environment should be avoided.

This article shows how to implement conventional page switching in conjunction with bit-plane layering on the IBM PC in the high-level $\mathrm{C}$ programming language. As 
such, it is hoped that these techniques will extend the research possibilities available to PC users.

\section{Availability}

Copies of the program can be obtained by e-mail to the author at bmyors@bunyip.bhs.mq.edu.au.

\section{REFERENCES}

BoкhoRst, F. D. (1995). Bit-plane layering for high-resolution EGA and VGA graphics on the IBM PC/XT/AT. Behavior Research Methods, Instruments, \& Computers, 27, 496-501.

FARRARo, R. (1994). Programmer's Guide to the EGA, VGA, and Super VGA Cards (3rd ed.). Reading, MA: Addison-Wesley.

FORSTER, K. I. (1970). Visual perception of rapidly presented word sequences of varying complexity. Perception \& Psychophysics, 8, 215221.

Graves, R. E., \& Bradley, R. (1991). Millisecond timing on the IBM $\mathrm{PC} / \mathrm{AT} / \mathrm{XT}$ and PS/2: A review of the options and corrections for the Graves and Bradley algorithm. Behavior Research Methods, Instruments, \& Computers, 23, 377-379.

Haussmann, R. E. (1992). Tachistoscopic presentation and millisecond timing on the IBM PC/XT/AT and PS/2: A Turbo Pascal unit to pro- vide general-purpose routines for CGA, Hercules, EGA, and VGA monitors. Behavior Research Methods, Instruments, \& Computers, 24, 303-310.

NETTLEBECK, T. (1987). Inspection time and intelligence. In P. A. Vernon (Ed.), Speed of information-processing and intelligence (pp. 295-346). Norwood, NJ: Ablex.

PotTER, M. C. (1993). Very short-term conceptual memory. Memory \& Cognition, 21, 156-161.

Raymond, J. E., Shapiro, K. L., \& Arnell, K. M. (1992). Temporary suppression of visual processing in an RSVP task: An attentional blink? Journal of Experimental Psychology: Human Perception \& Performance, 18, 849-860.

SARGENT, M., \& SHOEMAKER, R. L. (1995). The personal computer from the inside out (3rd ed.). Reading, MA: Addison-Wesley.

Segalowitz, S. J. (1987). IBM PC tachistoscope: Text stimuli. Behavior Research Methods, Instruments, \& Computers, 19, 383-388.

Wilton, R. (1994). Programmer's guide to PC video systems (2nd ed.). Redmond, WA: Microsoft Press.

\section{NOTE}

1. In this case mode 2 refers to programming counter 0 of the 8253 Programmable Interval Timer to mode 2 , not to a video mode. The mode 2 timer has a period of 838.1 nsec.

\section{LISTING}

Demonstration of the 32-Page Mode Odh Tachistoscope

// The PC tachistoscope has 32 pages.

\#include $<$ stdio. $\mathrm{h}>$

\#include $<$ conio.h $>$

\#include $<$ dos. $\mathrm{h}>$

\#include $<$ time.h $>$

\#include < graph.h>

\#define DISPLAY_WIDTH 320

\#define PAGE_SIZE $\quad 8192$

\#define ROM_8x8_FONT $\quad$ 0xf000fa6e

\#define ESCAPE $\quad 27$

\#define UP $\quad 72$

\#define DOWN $\quad 80$

// VGA control

\#define INPUT_STATUS_REGISTER $\quad 0 \times 3 \mathrm{da}$

\#define VSYNC_MASK

\#define ATTRIBUTE_CONTROLLER $\quad 0 \times 3 \mathrm{c} 0$

\#define COLOUR_PLANE_ENABLE $\quad 0 \times 12$

\#define RESET_ATTRIBUTE $\quad 0 \times 20$

\#define CRT_CONTROLLER $\quad 0 \times 3 \mathrm{~d} 4$

\#define CRT_ADDRESS_HI $\quad 0 \times 0 \mathrm{c}$

\#define CRT_ADDRESS_LO Ox0d

\#define SEQUENCER $\quad 0 \times 3 \mathrm{c} 4$

\#define MAP_MASK $\quad 0 \times 02$

// PROTOTYPES

void sync (void);

void set_page (int page);

void enable_plane (int bitplane);

void print_char (int row, int col, char thechar, char_far *vb);

void print_string (int row, int col, char *str, char_far *vb);

char_far $*$ video $=($ char_far $*) 0 x a 00000001 ; / /$ Graphics mode video buffer

void main (void) 


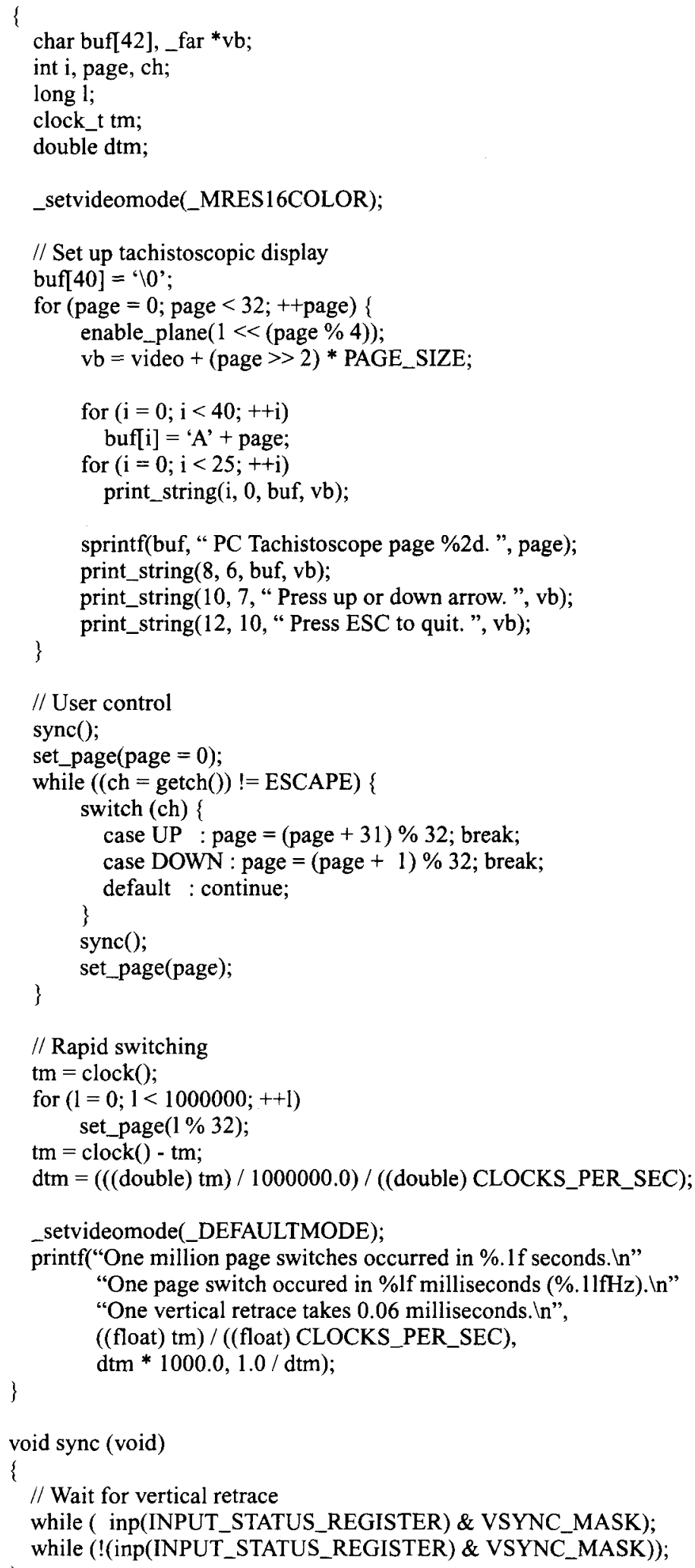




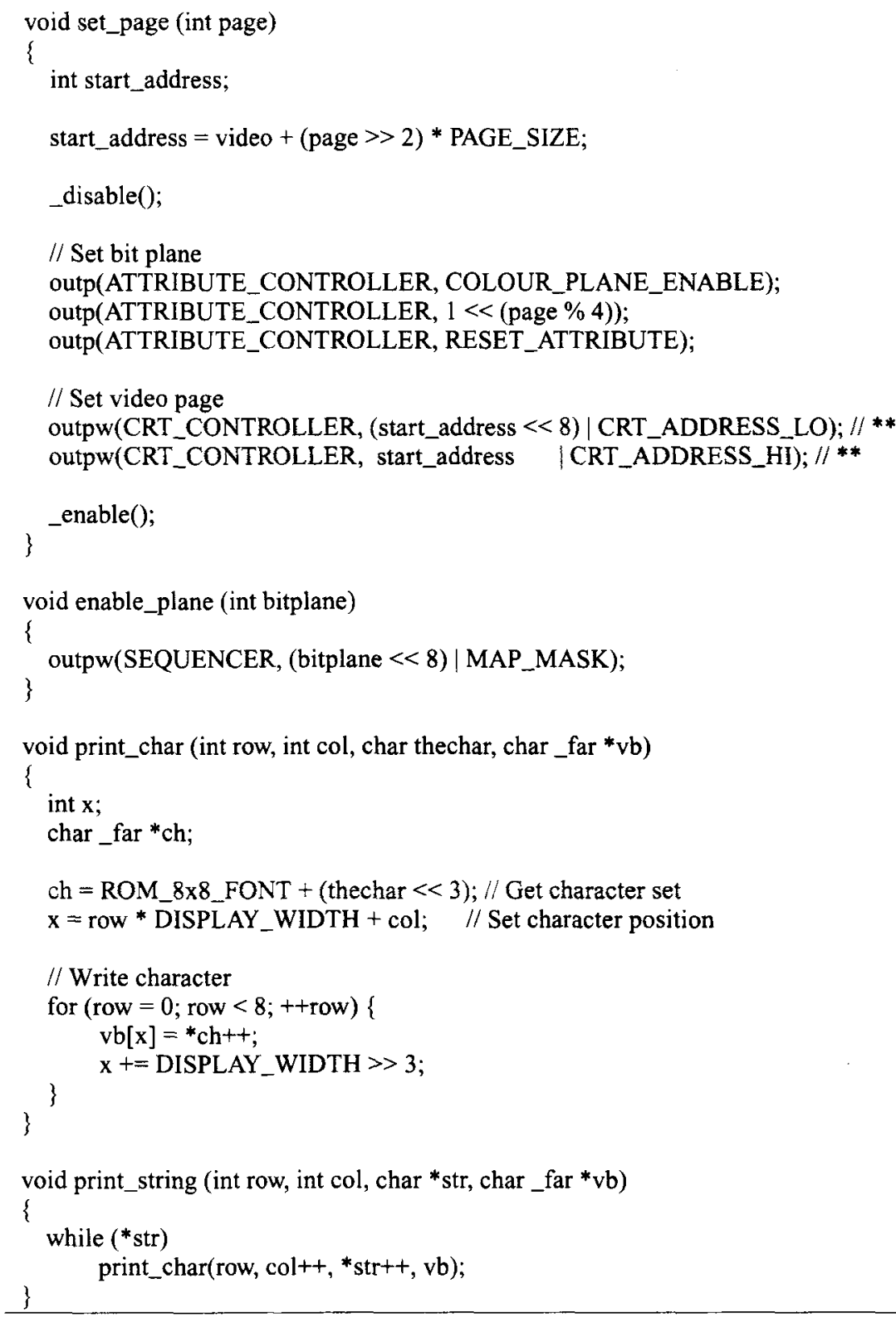

(Manuscript received January 15, 1997;

revision accepted for publication April 8, 1997.) 